\title{
What They Gain Depends on What They Do: An Exploratory Empirical Research on Effective Use of Mobile Healthcare Applications
}

\author{
Yao Yu \\ College of Management \\ and Economics, \\ Tianjin University, \\ Tianjin, China \\ yuyao_@tju.edu.cn
}

\author{
Xiangda Yan \\ JD Logistics, JD, \\ Beijing, China \\ yanxiangda1@jd.com
}

\author{
Xi Zhang \\ College of Management \\ and Economics, \\ Tianjin University, \\ Tianjin, China \\ jackyzhang@tju.edu.cn
}

\author{
Shuling Zhou \\ College of Management \\ and Economics, \\ Tianjin University, \\ Tianjin, China \\ zhoushuling@ @ tju.edu.cn
}

\begin{abstract}
Mobile healthcare application receives widespread attention, although it has advanced technology and user-friendly design, when users don't use it effectively, it seems worthless. Research mainly focus on technology improvement. But how to improve user behavior to match the technology is another essential factor for facilitating effective use from managerial perspective. We introduced the task technology fit theory to explain the mechanism when user using the applications. We added perceived e-health literacy as moderator variable, considering the user characteristic and medical environment. The data was collected from student samples of two schools (medical and nonmedical related universities), totally 178 valid samples. Our research indicates adaptation and learning behavior have significantly positive impact on the efficiency use and effectiveness use. The perceived ehealth literacy only has significant moderator effect on learning behavior. Our study provides practical implications for both software providers and users to achieve effective use of mobile healthcare applications.
\end{abstract}

\section{Introduction}

Mobile health is an up-to-the-moment medical mode with the development of medical industry and the innovation of information technology. It is defined as merging the mobile communications and information technology for healthcare [25]. Different from traditional medical software, mobile health provides health related applications at individual level. Due to the mobility and universality of the mobile medical devices, users can carry it and conduct health-related activities without time and space restrictions [26]. It has showed positive effects for health promotion [31, 43].

Mobile healthcare application has been on the rise for years. According to the "mHealth Economics program" , there are about 325 thousands health and fitness applications. In the same time, the number of these apps has nearly 50\% growth rate during 2016 to 2017 in Alphabet's Google Play store. Now, Alphabet's Google Play store has more health and fitness applications than iTunes, the number is 158 thousands and 155 thousands, respectively. Various mobile healthcare applications are in a period of rapid development, such as fitness apps, nutrition apps, selfcare apps, vitals apps, health information apps, etc. [11], proving that this field has received widespread attention. Despite of the rapid development and high download rate, many applications may not receive effective feedback, when users don't use it effectively. For example, many users have limited experience on using mobile applications, and their behavior habitat may not match the designed function. They just download it and try it several times, if it can't reach their expectation, then they delete it. In the consequence, despite there are more than 50 million apps uploaded, 95\% of them are abandoned, and these apps are called abandoned apps [20]. There are many issues causing the abandon. For example, the applications is crashed, the launch is too slow, or the function isn't as the user' expectation [3]. Recently, scholars have gradually concentrated on it. Countermeasures have also been proposed from the perspective of software attributes [28] and individual attributes [5]. The usability of the application play an important role on achieving effective use, which acquires the engineer to improve. But Perednia and Allen [38] argued that user's technology acceptance as a managerial challenge still plays an important role on the effectiveness of telemedicine, besides technology itself. So we argued the user behavior is a vital factor

\footnotetext{
1 https://research2guidance.com/product/mhealth-economics-2017current-status-and-future-trends-in-mobile-health/
} 
when accessing to the fresh application with advanced technology. Once the user can't adapt to the application, advanced technology and user-friendly interface seem worthless. So how to improve user behavior to match the technology is another essential perspective for effective use. After figuring out the mechanism from the perspective of the user behavior, it also becomes easier for the software provider to produce usability applications.

The existing research on effective use of mobile healthcare applications are relatively rare. Some scholars have explored the adoption mechanism of mobile healthcare applications [6], but there still exists gap that how the user behavior can improve the effective use. It is urgent to study the mechanism of effective use from user behavior perspective in the mobile healthcare applications environment, which can make up the defect that the user capabilities can't match the technology, and provide a new perspective for the software provider to improve application design from user behavior.

When using the mobile healthcare applications, users should plan for their goals, and be able to rationally express their aspirations based on the adaptation of new technology. Especially in medical field, due to the professional and medical attribution, users also need to have a certain level of e-health literacy to correctly understand the content and follow the instructions provided by the applications. Therefore, to explain the mechanism that how the user behavior influences the effective use of mobile healthcare applications, this study focuses on the task technology fit theory to figure out how the user adjustment affect the interaction among the task, technology and the user himself to achieve effective use. Meanwhile, we add the e-health literacy as moderator effect for additional explanation.

The contents of this paper are organized as follows: In literature review, we reviewed research objects and theories. Then we proposed research model and hypotheses. The process of data collection and data analysis are also presented. Finally, we discussed the theoretical and practical implication, and indicated the limitations and future work.

\section{Literature review}

\subsection{Mobile healthcare applications and effective use}

Mobile technology has contributed in various kinds of mobile health applications. For example, it can help assist the clinic with patient communication, disease treatment, and document management, etc. [46]. Mobile technology is also applied to special disease management, such as cancer [14], disability [2], and mental health [1]. It also helps in the area of chronic monitoring, such as monitoring, blood pressure and heart rate [32], prevent disease [13] and fitness management [7].

Scholars defined the effective use of information technology as the use behavior that the individuals use technology to accomplish the task successfully [12]. Burton-Jones et al. emphasized goal-directed attribution of effective use, and they proposed three components of the effective use, namely transparent interaction, representational fidelity, and informed action [12]. Transparent interaction refers to the degree that individual is unhampered when accessing both surface and deep structure of information technology. Representational fidelity refers to the degree that individual acquired result is exactly what the system represents. Informed action refers to the extent that individual act according to the exactly result obtained from system to improve their status. As for the mobile healthcare environment, when the user intends to improve health, how to state his illness, and which is the function to express his condition reflect the transparent interaction dimension. Then, the user gets the result of the consultation after expressing the need, like medical advice or instruction. The user knows where to get the correct feedback and has the ability to figure it out, which reflect representational fidelity. Finally, the user performs a series of actions, such as taking drugs based on the feedback from the software. It represents informed action. To sum up, in the mobile healthcare applications environment we regard the effective use as using the mobile healthcare applications to achieve goals of health improvement successfully. In general, the effective use of these three components ultimately affects the efficiency and effectiveness use of information technology [42]. Considering the goaldirected attribution, not only the properly diagnosis and treatment but also the timely prescription and medical advice are mandatory required when using the application for health improvement. So, not only the utility, but also the efficiency need to be considered to satisfy the users. Meanwhile, effectiveness and efficacy also contribute to enhance the performance of effective use in terms of goal achievement and return on investment [12]. So we explore the effective use of the mobile health application from two perspective, the effectiveness and the efficiency. The effectiveness use of the mobile application referred as using the mobile healthcare applications for health improvement is utility. The efficiency use of the mobile application referred as using the mobile healthcare application for health improvement is efficient.

The theoretical framework of effective use has been applied in various fields. In the term of medical and 
health technology, Assadi and Hassanein [6] applied the effective use into the research about use behavior of ehealth records, and noted that the effective use theory explained the improvement of individual readability on adoption behavior. Pare et al [37] proposed that effective use represents the use of "complete" or even "extended" for e-health records. Choi et al [15] explored the impact of the interface design of mobile healthcare applications on the effective use through comparison experiments. Weeger et al investigated the effective use in special clinical environments, and proposed current inaccuracies in medical technology [44].

\subsection{Task-technology fit theory}

The relationship between information technology and effective use is a concern for scholars. Goodhue and Thompson [24] first proposed technology-performance chain. They believed the performance of using information technology is closely related to the characteristics of task, technology, and individual. Any two parts of them are linked through the task technology fit theory (TTF). TTF refers to the degree that technology functionally match task requirements and individual capabilities [41]. Therefore, how to promote matching degree is the key element for achieving task technology fit, and it is considered as an important precondition for improving effective use [23].

Since the TTF was put forward, Goodhue [22] have further developed the theory, such as proposing the task technology fit measurement scales. And it also applied in different cultures across east and west cultures, like Chinese [29]. Delone and McLean [16] reviewed the previous information system model, and concluded that the task technology fit theory was complementary for information system from another perspective. Dishaw, and Strong [17] used task technology fit theory as an important variable to explore the impact on user acceptance, perceived ease use and perceived usefulness of information technology. Yen et al [45] applied the task technology fit theory to the adoption model of wireless devices and explored the impact of task characteristic and technical characteristics on task technology fit.

As users use the mobile healthcare applications, they participate in interaction among the technology, task and individual which are mentioned in the TTF. Technology is a tool for individuals to complete tasks which represents the application itself. Task refers to the individual behavior of converting input into output. In this study, it refers to the use of mobile healthcare applications to improve health. The individual is the basic element, and his characteristics will affect the use of task and technology.
To explore the user behavior, we summarized user behavior in mobile healthcare applications environment based on TTF. Barki et al [9] classified all the user behaviors of using information technology into three categories, namely technology interaction, tasktechnology adaptation and individual adaptation behavior. Technology interaction behavior represents all interactions that individuals perform information technology to accomplish tasks. The task-technology adaptation behavior refers to the behavior that they directly modify the technology. Individual adaptation behavior represents individual use technology through the promotion of their own capabilities. Among the three kinds of behaviors, the technology interaction behavior and task-technology adaptation behavior embody the adjustment for effective use between task and technology without individual changing. However, the individual adaptation behavior represents a form of learning behavior for effective use by improving the individual's capabilities without task and technology changed [4]. Therefore, in the mobile healthcare applications environment, we defined these two types of user behaviors as adaptation behavior and learning behavior, which can fully reflect the adjustment behaviors when using mobile health applications.

\subsection{Perceived electronic health literacy}

Aimed at improving e-health care, electronic health literacy represents the ability to get health information, and solve health problem via electronic sources [35]. Scholars proposed Lily model which divided e-health literacy into six dimensions, including traditional literacy, information literacy, media literacy, health literacy, computer literacy, and scientific literacy [35]. Norman and Skinner developed an e-health literacy scale for further study [34]. While they regarded ehealth literacy as an objective assessment indicator used to reflect how much performance users can receive through entire e-health activity. Considering the attributes of user self-perception, Zhang et al put forward the concept of perceived e-health literacy (PEHL) to figure out the users' continuance intention of mobile healthcare application and define it as the user's perceived ability to use information technology of mobile healthcare applications for improving health [47]. In this study, we selected perceived e-health literacy instead of e-health literacy to better understand the user behavior from the perspective of user perception. To emphasize the self-perceived attributes of perceived e-health, we introduce the concept of selfefficacy from the perspective of social cognitive to help understand the user behavior in mobile healthcare application. Self-efficacy determines individual's feeling, consideration, self-motivation and other 
behavior through four processes: cognition, motivation, emotion, and choice [8]. Research showed self-efficacy is a universal construct, claiming applicability across different cultures [39]. In the environment of using mobile healthcare applications, the cognitive process will promote the users to set higher goals about consulting the disease. The motivation process will motivate the users to pay more effort. The emotion process will help users to decrease the negative emotions and increase the confident to better use the application. The choice process will guide the users to choose more complex challenges.

\section{Hypotheses development}

To explore how the user behavior affects the effective use when using mobile healthcare applications, we introduced TTF to explain the interaction among the users, technology and task. We defined the performance of using mobile healthcare applications as efficiency use and effectiveness use of mobile healthcare application taking the utility and convenience into account. Considering the diversity of users' characteristics especially in medical environment, we focused on the perceived e-health literacy, and regarded it as moderator to figure out how the user behavior affects effective use with different level of perceived e-health literacy. The model of effective use of mobile healthcare applications is shown as Figure 1.

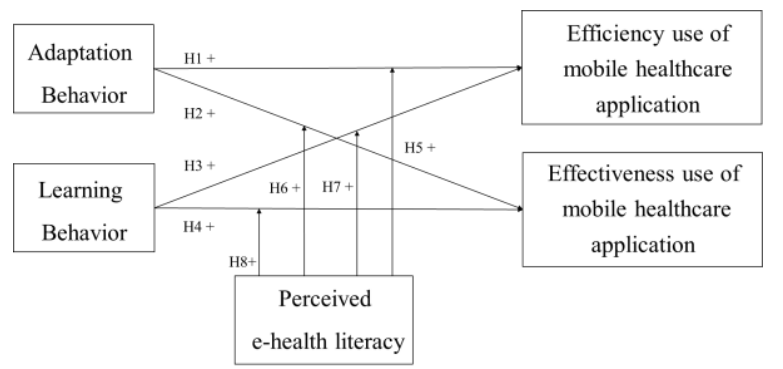

\section{Figure 1 Effective use behavior model of mobile healthcare applications}

\subsection{The impact of task-technology fit theory}

In this study, adaptation behavior refers to using information technology to achieve their goals by changing tasks or adapting technology. When users are seeking for health information, it requires them to have certain abilities to find what exactly they need. However, if users lack the ability, according to the task technology fit theory, they will adjust from two perspectives to achieve effective use [24]. One perspective is technology. For example, users can find the most accurate information refer to the view number of each post or the recommendation of the application. The advanced communication platform can significantly promote the task performance [10]. The other perspective is task, and users may adjust his own task. For example, they can change the structural task into less structural task so that the task performance can be improved [40]. According to the effective use framework, adaptation behavior can be explained by two dimensions of effective use, such as transparent interaction and informed action [12]. In the stage of task-technology adaptation, users interact with information. By modifying the use pattern of the system, they can quickly find the information they need from chaotic information. Therefore, individuals can gain more efficient and effective performance through the adaptation behavior of mobile healthcare applications. Therefore, we assume that,

H1. Adaptation behavior has a positive effect on the efficiency use of mobile healthcare applications.

H2. Adaptation behavior has a positive effect on the effectiveness use of mobile healthcare applications.

In this study, learning behavior refers to the behavior that users use information technology to achieve their goals by adjusting their own capabilities. That is to say, users want to find the health information they need exactly, but they do not have such capabilities. In addition to adjusting in terms of tasks and technology, users can adjust their own capabilities which is considered as learning behavior [9]. Users can adapt themselves to fit technology and tasks through learning process to achieve their goals. For example, users can learn how to verify which information is truly effective by consulting relevant experts. According to the effective use framework [12], we believe this behavior belongs to representational fidelity and informed action. When users learn knowledge and proficiency increases, users can select the accurate information more quickly. On the other hand, knowledge in related fields can help users to better understand information, and it is more conducive for users to verify truly useful information. In the consequence, it can help users to complete tasks more efficiently and effectively. So, we assume that,

H3. Learning behavior has a positive effect on the efficiency use of mobile healthcare applications.

H4. Learning behavior has a positive effect on the effectiveness use of mobile healthcare applications.

\subsection{Impact of perceived electronic health literacy on Effective Use}

As mentioned above, perceived e-health literacy can be understood by means of self-efficacy. Research showed that users take self-efficacy as consideration when they adapt mobile healthcare applications [33], and Liu [30] found that self-efficacy significantly affect the task technology fit process. When individual takes 
adaptation behavior from the perspective of tasks due to their strong cognition, they will reduce the task goal or convert it to other achievable goals. As individuals aimed to achieve their task, those with high levels of perceived e-health literacy will provide enough motivation for individuals to overcome technology difficulties due to their high level of self-efficacy. And they will spend more efforts to find adaptation methods [19]. Compared to individuals with low levels of perceived e-health literacy, they are more likely to obtain more effective use because they achieve their task or satisfy themselves. Therefore, we argue that individuals who possess high level of perceived e-health literacy will conduct adaptation behavior through the using technical functions and paying more efforts, so it will increase the efficiency use and effectiveness use. And the high level of e-health literacy will forest the effect of adaptation behavior. In summary, we assume that,

H5. Perceived e-health literacy positively moderates the effect of adaptation behavior on efficiency use of mobile healthcare applications.

H6. Perceived e-health literacy positively moderates the effect of adaptation behavior on effectiveness use of mobile healthcare applications.

On the other hand, individuals can achieve their goals for effective use as through self-enhancement such as learning behavior that we have hypothesized. When individuals have a high level of perceived e-health literacy, because of their high level of cognition, motivation, emotion and choice, they are willing to pay more effort to achieve the entire learning process. And Compared to individuals with low level of perceived ehealth literacy, they are more likely to achieve better learning outcomes, and their capabilities can be further improved to fit the task and technology. Therefore, we believe individuals with higher level of perceived ehealth literacy are more likely to obtain effective use through learning behavior, as the high level of e-health literacy makes individuals to spend less effort and complete the entire learning process faster to achieve the goal. Therefore, we assume that,

H7. Perceived e-health literacy positively moderates the effect of learning behavior on efficiency use of mobile healthcare applications.

H8. Perceived e-health literacy positively moderates the effect of learning behavior on effectiveness use of mobile healthcare applications.

\section{Data collection and analysis}

\subsection{Measurement}

In this study, the measurements were developed from prior research. 7-Point Likert-type scale was used to measure the variables. Efficiency use, effectiveness use, adaptation behavior, learning behavior and perceived e-health literacy were measured. The measurement of the effectiveness use (EF) was modified from Burton-Jones et al. [12], and efficiency use (EI) was based on Gattiker et al. [21]. The measurement of adaptation behavior (AB) and learning behavior (LB) related to task technology fit is based on Barki et al. [9]. The measurement of perceived e-health literacy (PEHL) was referenced by Norman and Skinner [34].

To ensure the validity of the measurement, the items were translated into Chinese first, and then translated back into English by the researchers to confirm the Chinese version was consistent with the English version. All the researchers have relevant information technology experience and expertise in English translation. Specifically, we revised the measurement based on mobile healthcare applications environment. Preliminary tests were also conducted by 10 users with experience in using mobile healthcare applications. In order to better ensure the validity of the questionnaire, we adapted it with the suggestions provided by the preliminary test users and modified some unclear and ambiguous questions to make sure the questionnaire is clarity.

\subsection{Data collection}

Our data was collected from student samples. Norman and Skinner hypothesized that e-health literacy is related to technology use [35]. University students can access to mobile technology more often, especially on internet use [27]. So, the student sample is an ideal data source in this study. The college students are chosen from both medical-related schools and non-medical related schools. The perceived e-health literacy of the tow student groups is different because students in medical-related schools acquire more health knowledge through education. Therefore, they may have relatively high perceived e-health literacy. However, students in non-medical related schools usually do not learn about healthcare, so their perceived e-health literacy is relatively low. So, the level of perceived e-health literacy is diverse for data analysis.

In addition, as university students use mobile applications more frequently, the students group is also familiar to the mobile health application. Besides the long term diseases, students also use mobile health application to manage other health problems. Such as, they often use fitness app to keep fit and sleep apps to manage sleep quantity. Thus, it made the college students become a reliable group for us to explore the effective use mechanism of mobile healthcare applications. It is also worth noting that Chinese students cannot register as doctors in mobile healthcare 
applications, which ensures that all samples are mobile healthcare applications users instead of health service providers. To further ensure that samples are users of mobile applications, the questionnaire is collected online by WeChat (a popular social media in China).

The process of questionnaire distribution was divided into two stages. In the first stage, we chose 4 students in medical-related schools and 3 students in non-medical-related schools. In order to have a better questionnaire, we used the one-to-one questionnaire to collect more samples in the second stage. In this way, a total of 104 questionnaires were sent to medical-related schools, and 99 questionnaires were sent to nonmedical-related schools. 97 medical-related individuals and 81 non-medical related individuals responded to our questionnaire. We used three criteria to filter valid questionnaires, including whether the questionnaire had missing values on some data (deletion of missing data), whether the questionnaire was submitted within 20 seconds (delete data within 20 seconds) and whether the respondent have experience in using mobile healthcare applications (delete inexperienced data). Finally, 178 valid sample with an average response time of 78.2 seconds (S.D. =36.3) were collected.

\subsection{Data analysis}

This study used partial least squares (PLS) for data analysis. The PLS is suitable for small and medium samples [30]. In the specific analysis process, we conducted the SmartPLS 3.2.7. We collected 178 valid data, exceeding the sample requirement for the largest multiple, which shows that our sample is in accordance with the rules of thumb, so we can conduct the SEM analysis. The analysis of the SEM proceeds from two aspects. The first aspect is to measure the model, including the analysis of reliability and validity. The second aspect is the analysis of the path coefficient and significance of the model, which is used to judge whether the theoretical hypothesis is supported.

\section{Results}

\subsection{Demographic Analysis}

The statistical results of demographic data are shown in Table 1. Including 96 males and 82 females. 178 participants were aged from 20 to 29 , accounting for $95.5 \%$. There were 97 medical-related $(54.49 \%)$ and 81non-medical-related participants (45.50\%). The average PEHL level for medical-related participants was 4.84 (S.D. = 0.937), and non-medical-related participants was $4.43($ S.D. $=0.625)$.

\subsection{Measurement model}

The test of measurement model is conducted by SmartPLS 3.2.7. Quality criteria results are shown in Table 2. All the average variance extracted (AVE) are all bigger than 0.5 , Composite Reliability (CR) and Cronbach's alpha $(\mathrm{C} \alpha)$ are over 0.7 . All the indicators shows good reliability [36]. Meanwhile we conducted the Fornell-Larcker Criterion (FLC) and CrossLoadings (CLs) to measure discriminant validity. Specific data are shown in Tables 3 and 4. The AVE square root of the variable exceed its correlation coefficients with other variables. The results show our study has good validity [18].

Table 1. Demographic statistics

\begin{tabular}{|c|c|c|}
\hline & Item & Percentage \\
\hline \multirow[t]{2}{*}{ Gender } & male & $96(53.9 \%)$ \\
\hline & female & $82(46.1 \%)$ \\
\hline \multirow[t]{2}{*}{ Age } & $20-29$ & $170(95.5 \%)$ \\
\hline & $30-39$ & $8(4.5 \%)$ \\
\hline \multicolumn{3}{|c|}{ PEHL levels with medical-related, non-medical } \\
\hline \multirow{3}{*}{$\begin{array}{c}\text { medical-related } \\
\text { non-medical }\end{array}$} & $\begin{array}{c}\text { Amount } \\
\text { (percentage) }\end{array}$ & $\begin{array}{c}\text { PEHL average } \\
(\mathrm{SD})\end{array}$ \\
\hline & $97(54.49 \%)$ & $4.84(0.937)$ \\
\hline & $81(45.50 \%)$ & $4.43(0.625)$ \\
\hline
\end{tabular}

Table 2. Quality criteria

\begin{tabular}{cccc}
\hline Measurement variable & AVE & CR & Co \\
\hline AB & 0.824 & 0.949 & 0.929 \\
LB & 0.814 & 0.946 & 0.924 \\
PEHL & 0.818 & 0.969 & 0.963 \\
EI & 0.924 & 0.973 & 0.959 \\
EF & 0.927 & 0.974 & 0.961 \\
\hline
\end{tabular}

Table 3. Fornell-Larcker Criterion

\begin{tabular}{cccccc}
\hline $\begin{array}{c}\text { Measurement } \\
\text { variable }\end{array}$ & AB & EF & EI & LB & PEHL \\
\hline AB & $\underline{\mathbf{0 . 9 0 8}}$ & & & & \\
EF & 0.774 & $\underline{\mathbf{0 . 9 6 3}}$ & & & \\
EI & 0.798 & 0.941 & $\underline{\mathbf{0 . 9 6 1}}$ & & \\
LB & 0.119 & 0.591 & 0.555 & $\underline{\mathbf{0 . 9 0 2}}$ & \\
PEHL & 0.946 & 0.779 & 0.804 & $\underline{0.093}$ & $\underline{\mathbf{0 . 9 0 4}}$ \\
\hline
\end{tabular}

\subsection{Structural model}

Based on the reliability and validity of the model, we analyze the coefficient and significant. We applied SmartPLS 3.2.7 to analysis the structural model as well as the moderator effect. The bootstrapping was set 5000 . The results indicate that the $\mathrm{R}^{2}$ of the efficiency use and effectiveness use are 0.888 and 0.889 , respectively. It shows that the model has a good explanation. The analysis result of the path coefficients shows that six 
hypotheses are supported. While H5 and H6 are not significantly supported. The specific research and analysis results are shown in Table 5 and displayed in Figure 2.

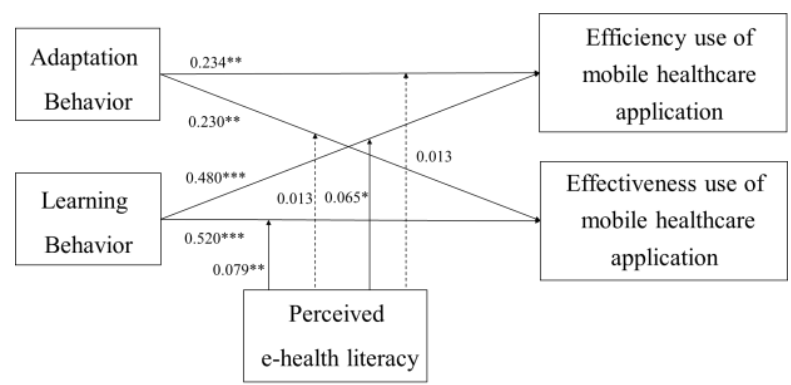

Figure 2 Result of structural model

\section{Discussion and contributions}

In this study, we explain the mechanism how the user behavior affects their effective use of the mobile healthcare applications. Our research indicated individuals can adjust tasks, the use of technology, and their abilities as well as enhance the perceived e-health literacy to improve effective use. More specifically, the adaptation behavior and the learning behavior have a significantly positive impact on the efficiency use and effectiveness use of the mobile healthcare applications. It is worth noting that the coefficient and P-value of the learning behavior are bigger than the adaptation behavior, which shows that learning behavior has more important impact compared with adaptation behavior. We believe that learning behavior is a more stable way to increase the effective use. Because the technology of mobile healthcare applications develops too fast, and the functions of the mobile healthcare applications are changing over time, the user adaptation behavior by adjusting task and technology must be changed all the time. Obviously, learning behavior can substantially enhance the individual's ability to fit the changing function with advanced technology, so the learning behavior have stronger effect.

To explore the impact of users' characteristics on user behavior, we verify the influence of perceived ehealth literacy. The result indicated it can enhance the individuals' adjustment pattern to achieve effective use. Specifically, perceived e-health literacy has the significant moderator effect on learning behavior instead of the adaptation behavior. As the hypothesis has stated that perceived e-health literacy will facilitate the learning behavior by motivating users to pay more efforts to improve individual capabilities. However, the adaptation behavior is not moderated by the perceived e-health literacy, though the technology adaptation can improve the individual's effective use without significantly reducing the user's goal as hypothesized. The great reliance on technical capabilities may allow individuals to choose complex processes even wrong processes based on the high level of perceived e-health literacy, which will finally lead to a negative impact on effective use. Therefore, we believe that the complexity of technical functions are the reasons why these hypotheses are not significantly supported.

\subsection{Theoretical implications}

This study has many important theoretical contributions. First, in the field of mobile healthcare applications, there are few research focus on how user behavior influences the effective use. Although some research explored the impact of user behavior on the use intention, they haven't underlined the mechanism of effective use from the user behavior perspective. We carried out the TTF to explain the impact mechanism of user behavior on effective use, which enriched the mobile healthcare applications research field from theoretical perspective.

Second, since the concept of perceived e-health literacy was put forward, more and more research has emerged, but the existing research merely focused on using this variable to measure perceived e-health literacy level of different groups and explored way to improve the relevant abilities. Only few studies applied e-health literacy as a driver. In this study, we considered perceived e-health literacy as an important factor in moderating the match between user characteristic and information technology of mobile healthcare applications, and it provided a new research perspective for future research.

\subsection{Practical implication}

Our results also contributed to some practical implication not only for mobile healthcare applications providers from managerial perspective, but also for users to improve effective use. We believe that software providers should improve the usability of application from two perspectives. Firstly, from the perspective of technical functions, software providers should design more convenient functions to match the users' technology skill level, which can make it easier for users to obtain effective use when they conduct adaptation behaviors. In addition, the results of the study also show that individuals with different levels of perceived ehealth literacy have different effects on effective use. So, to improve the usability for different user group software providers should identify different users' ehealth literacy level firstly, and then adopt the targeted 
Table 4. Cross-loadings

\begin{tabular}{|c|c|c|c|c|c|c|}
\hline $\begin{array}{l}\text { Measurement } \\
\text { variable }\end{array}$ & Measurement items & $\mathbf{A B}$ & $\mathbf{L B}$ & PEHL & EI & $\mathbf{E F}$ \\
\hline \multirow{5}{*}{$\mathrm{AB}$} & AB_1 & $\underline{0.922}$ & 0.093 & 0.857 & 0.715 & 0.692 \\
\hline & $\mathrm{AB} \_2$ & $\overline{0.898}$ & 0.135 & 0.841 & 0.731 & 0.703 \\
\hline & AB_3 & $\overline{0.901}$ & 0.080 & 0.869 & 0.711 & 0.682 \\
\hline & AB_4 & $\underline{0.909}$ & 0.122 & 0.867 & 0.739 & 0.732 \\
\hline & LB_-1 & $\overline{0.118}$ & $\underline{0.905}$ & 0.107 & 0.527 & 0.531 \\
\hline \multirow{3}{*}{ LB } & LB_2 & 0.091 & $\overline{0.910}$ & 0.053 & 0.494 & 0.538 \\
\hline & LB_3 & 0.141 & $\overline{0.904}$ & 0.132 & 0.531 & 0.580 \\
\hline & LB_4 & 0.072 & $\overline{0.892}$ & 0.035 & 0.442 & 0.477 \\
\hline \multirow{7}{*}{ PEHL } & PEHL_1 & 0.846 & 0.074 & $\underline{0.897}$ & 0.701 & 0.684 \\
\hline & PEHL_2 & 0.872 & 0.034 & $\overline{0.918}$ & 0.743 & 0.704 \\
\hline & PEHL_3 & 0.860 & 0.088 & $\overline{0.909}$ & 0.735 & 0.715 \\
\hline & PEHL_4 & 0.836 & 0.072 & $\overline{0.898}$ & 0.724 & 0.706 \\
\hline & PEHL_5 & 0.850 & 0.116 & $\overline{0.892}$ & 0.729 & 0.711 \\
\hline & PEHL_6 & 0.874 & 0.121 & $\overline{0.910}$ & 0.737 & 0.721 \\
\hline & PEHL_7 & 0.846 & 0.084 & $\overline{0.906}$ & 0.720 & 0.688 \\
\hline \multirow{3}{*}{ EI } & EI_1 & 0.799 & 0.505 & 0.809 & $\underline{0.968}$ & 0.913 \\
\hline & EI_2 & 0.749 & 0.536 & 0.754 & $\underline{0.959}$ & 0.897 \\
\hline & EI_3 & 0.752 & 0.560 & 0.756 & $\overline{0.957}$ & 0.905 \\
\hline \multirow{3}{*}{$\mathrm{EF}$} & EF_1 & 0.744 & 0.569 & 0.752 & $\overline{0.911}$ & $\underline{0.969}$ \\
\hline & EF_2 & 0.727 & 0.588 & 0.743 & 0.909 & $\overline{0.964}$ \\
\hline & EF_3 & 0.765 & 0.551 & 0.754 & 0.898 & $\underline{0.955}$ \\
\hline
\end{tabular}

Table 5. Analysis results of structural equation model

\begin{tabular}{clccc}
\hline \multicolumn{1}{c}{ Hypothesis } & Coefficient & $\begin{array}{c}\text { P- } \\
\text { value }\end{array}$ & $\begin{array}{c}\text { Support } \\
\text { or not }\end{array}$ \\
\hline $\mathrm{H} 1+\begin{array}{l}\text { Adaptation behavior has a positive effect on the efficiency use of mobile } \\
\text { healthcare applications. }\end{array}$ & $0.234^{* *}$ & 0.006 & $\sqrt{ }$ \\
$\mathrm{H} 2+\quad \begin{array}{l}\text { Adaptation behavior has a positive effect on the effective use of mobile } \\
\text { healthcare applications. }\end{array}$ & $0.230^{* *}$ & 0.005 & $\sqrt{ }$ \\
$\mathrm{H} 3+\quad \begin{array}{l}\text { Learning behavior has a positive effect on the efficiency use of mobile } \\
\text { healthcare applications }\end{array}$ & $0.480^{* * *}$ & 0.000 & $\sqrt{ }$ \\
$\mathrm{H} 4+\quad \begin{array}{l}\text { Learning behavior has a positive effect on the effective use of mobile } \\
\text { healthcare applications. }\end{array}$ & $0.520^{* * *}$ & 0.000 & $\sqrt{ }$ \\
$\mathrm{H} 5+\quad \begin{array}{l}\text { Perceived e-health literacy positively moderates the effect of adaptation } \\
\text { behavior on efficiency use of mobile healthcare applications. }\end{array}$ & 0.013 & 0.635 & $\times$ \\
$\mathrm{H} 6+\quad \begin{array}{l}\text { Perceived e-health literacy positively moderates the effect of adaptation } \\
\text { behavior on effective use of mobile healthcare applications. }\end{array}$ & 0.013 & 0.497 & $\times$ \\
$\mathrm{H} 7+\quad \begin{array}{l}\text { Perceived e-health literacy positively moderates the effect of learning } \\
\text { behavior on efficiency use of mobile healthcare applications. }\end{array}$ & $0.065^{*}$ & 0.026 & $\sqrt{ }$ \\
$\mathrm{H} 8+\quad \begin{array}{l}\text { Perceived e-health literacy positively moderates the effect of learning } \\
\text { behavior on effective use of mobile healthcare applications. }\end{array}$ & $0.079^{* *}$ & 0.004 & $\sqrt{ }$ \\
\hline
\end{tabular}

Note: ${ }^{*}=p<.05 ;{ }^{* *}=p<.01 ;{ }^{* * *}=p<.001$

strategies to display different functions and technology according to the users' level of perceived e-health literacy. For example, for the elderly group with low level of perceived e-health literacy, the application is supposed to provide common encapsulated service with convenience interface. As for the group with high level of perceived e-health literacy, the application can display complex functions, like personalize search and artificial intelligence functions. Secondly, from the user perspective, software providers should provide users with simple instructive information on the startup interface, such as instruction manual of the software. It can provide a better and more effective way for users to learn and get familiar with the applications.

Besides improving the usability of the application, there are some practical implications for users to improve their behavior. In the process of achieving 
effective use, our research indicated that the learning behavior has stronger impact compared to the adaptation behavior, so the users are supposed to improve their capabilities to find the best method to using the application effectively. Meanwhile, the users also need to spend some time to get accustomed to the application and know about the functions instead of superficial trying. They also need to attempt to convert their goals into realizable aims which can be solved by the application easily. What's more, if users want to use the mobile healthcare application to manage health themselves, it is urgent to improve their perceived ehealth literacy level. On the one hand, it can promote the user strengthening the learning behavior. On the other hand, it can enhance user capabilities to keep pace with the changing technology of the mobile healthcare application to achieve effective use.

\section{Limitation}

First, our research only studied the university student group. It is our initial phase to work out the mechanism of the effective use of mobile healthcare applications. As the aging problem becomes more and more serious, one of the most urgent tasks of mobile healthcare applications is to solve the health problems of the elderly. Therefore, based on this research, we will focus on the target group of elderly people with low perceived e-health literacy and serious disease for further research.

Second, the sample of this study was collected through questionnaire, which is inevitably subjective, and might lead to comprehending deviation. So the result of discriminant validity is less than satisfactory due to the concept similarity of the measurement. Future research should consider other data collection and analysis methods, such as data mining to avoid comprehending deviation and promote data quality.

Finally, the research considered the software as the technology itself, regardless the specific function. To further explore how the user behavior can match the technology of the mobile healthcare applications, future work can focus on the interaction between the users and the specific functions.

\section{Acknowledgement}

The work described in this study was supported by National Natural Science Foundation of China (71571133).

\section{References}

[1] Abu Rahal, Z., Vadas, L., Manor, I., Bloch, B. and Avital, A. Use of information and communication technologies among individuals with and without serious mental illness. Psychiatry Research, 266(Aug 2018), 160-167.

[2] Agarwal, Y. Accessibility information in New Delhi for "EasenAccess" Android-based app for persons with disability: an observational study. Disability and rehabilitation. Assistive technology(2018-Jun-14 2018), 118.

[3] Ajzen, I. The theory of planned behavior. Organizational Behavior \& Human Decision Processes, 50, 2 1991), 179211.

[4] Ammenwerth, E., Iller, C. and Mahler, C. IT-adoption and the interaction of task, technology and individuals: a fit framework and a case study. BMC medical informatics and decision making, 6(2006 Jan 2006), 3-3.

[5] Angst, C. M. and Agarwal, R. Adoption of Electronic Health Records in the Presence of Privacy Concerns: The Elaboration Likelihood Model and Individual Persuasion. Mis Quarterly, 33, 2 2009), 339-370.

[6] Assadi, V. and Hassanein, K. Consumer Adoption of Personal Health Record Systems: A Self-Determination Theory Perspective. Journal of Medical Internet Research, 19, 7 (Jul 2017).

[7] Azar, K. M. J., Bennett, G. G., Nolting, L. A., Rosas, L. G., Burke, L. E. and Ma, J. A framework for examining the function of digital health technologies for weight management. Translational Behavioral Medicine, 8, 2 (Apr 2018), 280-294.

[8] Bandura, A. Self-efficacy: The exercise of control. Journal of Cognitive Psychotherapy, 13, 2 2005), -.

[9] Barki, H., Titah, R. and Boffo, C. Information System Use-Related Activity: An Expanded Behavioral Conceptualization of Individual-Level Information System Use. Information Systems Research, 18, 2 2007), 173-192.

[10] Belanger, F., Collins, R. W. and Cheney, P. H. Technology Requirements and Work Group Communication for Telecommuters. Information Systems Research, 12, 2 2001), 155-176.

[11] Bol, N., Helberger, N. and Weert, J. C. M. Differences in mobile health app use: A source of new digital inequalities? Information Society, 34, 3 (2018 2018), 183-193.

[12] Burton-Jones, A. and Grange, C. From Use to Effective Use: A Representation Theory Perspective. Information Systems Research, 24, 3 (Sep 2013), 632-658.

[13] Cella, M., Okruszek, L., Lawrence, M., Zarlenga, V., $\mathrm{He}, \mathrm{Z}$. and Wykes, T. Using wearable technology to detect the autonomic signature of illness severity in schizophrenia. Schizophrenia Research, 195(May 2018), 537-542.

[14] Choi, J., Cho, Y. and Woo, H. mHealth Approaches in Managing Skin Cancer: Systematic Review of EvidenceBased Research Using Integrative Mapping. Jmir Mhealth and Uhealth, 6, 8 (Aug 2018).

[15] Choi, W. and Tulu, B. Effective Use of User Interface and User Experience in an mHealth Application. City, 2017. [16] DeLone, W. H. and McLean, E. R. The DeLone and McLean model of information systems success: a ten-year update. Journal of Management Information Systems, 19, 4 (Spr 2003), 9-30.

[17] Dishaw, M. T. and Strong, D. M. Extending the technology acceptance model with task-technology fit 
constructs. Information \& Management, 36, 1 (Jul 1999), 921.

[18] Fornell, C. and Larcker, D. F. Evaluating Structural Equation Models with Unobservable Variables and Measurement Error. Journal of Marketing Research, 18, 1 1981), 39-50.

[19] Gao, Y., Li, H. and Luo, Y. An empirical study of wearable technology acceptance in healthcare. Industrial Management \& Data Systems, 115, 9 (2015 2015), 17041723.

[20] Gary, R. and Care, M. NUCC1311 InSight_Why Mobile Apps Abandoned 122011.

[21] Gattiker, T. F. and Goodhue, D. L. What Happens After ERP Implementation: Understanding the Impact of Interdependence and Differentiation on Plant-Level Outcomes. City, 2005.

[22] Goodhue, D. L. Development and Measurement Validity of a Task - Technology Fit Instrument for User Evaluations of Information System. Decision Sciences, 29, 1 2010), 105-138.

[23] Goodhue, D. L., Klein, B. D. and March, S. T. User evaluations of IS as surrogates for objective performance. Information \& Management, 38, 2 (Dec 2000), 87-101.

[24] Goodhue, D. L. and Thompson, R. L. Task-Technology Fit and Individual Performance. MIS Quarterly, 19, 2 1995), 213-236.

[25] Istepanian, R. S. H., Jovanov, E. and Zhang, Y. T. Introduction to the special section on m-Health: Beyond seamless mobility and global wireless health-care connectivity. Ieee Transactions on Information Technology in Biomedicine, 8, 4 (Dec 2004), 405-414.

[26] Kafeza, E., Chiu, D. K. W., Cheung, S. C. and Kafeza, M. Alerts in mobile healthcare applications: Requirements and pilot study. Ieee Transactions on Information Technology in Biomedicine, 8, 2 (Jun 2004), 173-181.

[27] Kvavik, R. Convenience, Communications, and Control: How Students Use Technology. City, 2005.

[28] Lauterbach, J., Kahrau, F., Mueller, B. and Maedche, A. What makes " the System " tick ? - Explaining Individuals' Adaptation Behavior towards Effective Use in Enterprise System Implementations2014).

[29] Lin, W. S. and Wang, C. H. Antecedences to continued intentions of adopting e-learning system in blended learning instruction: A contingency framework based on models of information system success and task-technology fit. Computers \& Education, 58, 1 2012), 88-99.

[30] Liu, Z., Min, Q. and Ji, S. An empirical study of mobile securities management systems adoption: a TaskTechnology Fit perspective. International Journal of Mobile Communications, 8, 2 (2010 2010), 230-243.

[31] Martinez-Perez, B., de la Torre-Diez, I. and LopezCoronado, M. Mobile Health Applications for the Most Prevalent Conditions by the World Health Organization: Review and Analysis. Journal of Medical Internet Research, 15, 6 (Jun 2013).

[32] Modena, B. D., Bellahsen, O., Nikzad, N., Chieh, A., Parikh, N., Dufek, D. M., Ebner, G., Topol, E. J. and Steinhubl, S. Advanced and Accurate Mobile Health Tracking Devices Record New Cardiac Vital Signs. Hypertension, 72, 2 (Aug 2018), 503-510.
[33] Mohamed, A. H. H. M., Tawfik, H., Al-Jumeily, D. and Norton, L. MoHTAM: A Technology Acceptance Model for Mobile Health Applications. City, 2011.

[34] Norman, C. D. and Skinner, H. A. eHEALS: The eHealth Literacy Scale. Journal of Medical Internet Research, 8, 4 (Oct-Dec 2006).

[35] Norman, C. D. and Skinner, H. A. eHealth literacy: Essential skills for consumer health in a networked world. Journal of Medical Internet Research, 8, 2 (2006 2006).

[36] Nunnally, J. C. Psychometric theory. American Educational Research Journal, 5, 3 1978), 83.

[37] Pare, G., Raymond, L., de Guinea, A. O., Poba-Nzaou, P., Trudel, M.-C., Marsan, J. and Micheneau, T. Electronic health record usage behaviors in primary care medical practices: A survey of family physicians in Canada. International Journal of Medical Informatics, 84, 10 (Oct 2015), 857-867.

[38] Perednia, D. A. and Allen, A. Telemedicine technology and clinical applications. Jama, 273, 6 (1995-Feb-08 1995), 483-488.

[39] Schwarzer, R., Bäßler, J., Kwiatek, P., Schröder, K. and Zhang, J. X. The Assessment of Optimistic Self-beliefs: Comparison of the German, Spanish, and Chinese Versions of the General Self-efficacy Scale. Applied Psychology, 46, 1 1997), 69-88.

[40] Shirani, A. I., Tafti, M. H. A. and Affisco, J. F. Task and technology fit: a comparison of two technologies for synchronous and asynchronous group communication. Information \& Management, 36, 3 (Sep 1999), 139-150.

[41] Sun, H. and Zhang, P. Adaptive System Use; An Investigation at the System Feature Level. City, 2008.

[42] Tanja, G. and Jurij, J. Three Dimensions of Business Intelligence Systems Use Behavior. International Journal of Enterprise Information Systems (IJEIS), 10, 3 2014), 62-76. [43] Valdivieso-Lopez, E., Flores-Mateo, G., MolinaGomez, J.-D., Rey-Renones, C., Barrera Uriarte, M.-L., Duch, J. and Valverde, A. Efficacy of a mobile application for smoking cessation in young people: study protocol for a clustered, randomized trial. Bmc Public Health, 13(Aug 1 2013).

[44] Weeger, A., Neff, A., Gewald, H. and Haase, U. Exploring Determinants of Effective Use: The Role of Misfits between a Hospital and its Information Systems. City, 2014. [45] Yen, D. C., Wu, C.-S., Cheng, F.-F. and Huang, Y.-W. Determinants of users' intention to adopt wireless technology: An empirical study by integrating TTF with TAM. Computers in Human Behavior, 26, 5 (Sep 2010), 906-915.

[46] Zeman, J. E., Moon, P. S., McMahon, M. J. and Holley, A. B. Developing a Mobile Health Application to Assist With Clinic Flow, Documentation, Billing, and Research in a Specialty Clinic. Chest, 154, 2 (Aug 2018), 440-447.

[47] Zhang, X., Yan, X., Cao, X., Sun, Y., Chen, H. and She, J. The role of perceived e-health literacy in users' continuance intention to use mobile healthcare applications: an exploratory empirical study in China. Information Technology for Development, 2 2016), 1-26. 